\title{
Analysis of a Novel SPS Configuration Enabled by Lunar ISRU
}

\author{
Peter J. Schubert, Ph.D., P.E., Sheylla Monteiro Pinto, Bruna Caroline Pires, Moises do Nascimento, Edward Barks, \\ Jonathan Nderitu, Gabriel Goncalves, Fatih Tokmo \\ IUPUI, Indianapolis, IN 46202
}

\begin{abstract}
Architectures for space-based solar power using in situ resource utilization (ISRU) of space materials can greatly reduce earth launch mass and can enable geometric capacity growth. These two factors allow the potential for low cost power generation after development of an in-space infrastructure. A collection of extraction and processing methods designed for lunar operation provides for large volumes of low cost solar panels. With abundant panels a novel configuration for solar power satellites (SPS) is possible which avoids many of the challenges of existing designs. The so-called "tin can" SPS has no moving parts. It includes integral thermal radiators. Station-keeping requirements are minimal. Structural integrity is designed-in so that balance of plant mass is minimal. In this work the architecture and infrastructure supporting the tin can SPS is developed to support rapid construction and deployment. Performance estimates for the SPS are provided regarding heat and energy balance, and specific mass requirements.
\end{abstract}

\section{Introduction}

$\mathrm{E}$ nergy generation technologies vary in their up-front capital costs and in on-going expenses to purchase fuel, maintain operations, and conduct maintenance. Over the expected lifetime such assets will produce power according to their up-time. The ratio of total costs to total power produced is a metric called Levelized Cost of Energy (LCOE) and is expressed in units of US dollars per megaWatt-hour ( $\$ / \mathrm{MWh})$. LCOE represents the breakeven price of electricity on a wholesale market. The 2015 US average price is about $\$ 37 / \mathrm{MWh}^{1}$, which includes a blend of generation technologies, and a mix of new installations and legacy power stations, some of which are 50 years old. LCOE is most useful when comparing new utility-scale installations purely on price. LCOE for wind and solar are becoming competitive with conventional power generation largely because they have zero fuel costs. However, this metric does not account for availability of power, which can be a significant factor when considering terrestrial-based renewable sources such as wind and solar - which are intermittent in ways that are beyond human control. Space Solar Power is a form of renewable energy intended to address intermittency of terrestrial renewables by providing baseload ("always on") power. If fabricated on a sufficiently large scale, SSP has the potential for energy which is low in cost, and low in environmental pollution on a per megawatt-hour basis.

The physics of satellite launch work against SSP in that enormous costs are incurred to boost the needed hardware into orbit for building solar power satellites (SPS). Architectures for building and operating SPSs from materials already in space have the advantage of reducing launch costs, and the further advantage of moving most manufacturing operations off-planet where pollution concerns are ameliorated. With in-space factories that consume space minerals and produce solar panels and structural materials, the amount of mass launched from earth is reduced. For instance a 1.3 metric ton (MT) factory launched to the lunar poles can convert powdered regolith into 64 MT of pure oxygen each year ${ }^{2}$. A similar quantity of silicon can also be produced, fashioned into slabs, and processed into photovoltaic (PV) cells ${ }^{3}$. Launch into orbit requires 24 times less energy when starting from the moon, and benefits further from the lack of a friction-generating atmosphere. As such, it is possible to fabricate PV panels on the moon and deliver them electromagnetically into orbit where they can be electromagnetically received ${ }^{4}$. This makes feasible new configurations for SPSs which overcome several of the key technical challenges experienced by previous SSP architectures. In this work, it is assumed that lunar-sourced PV panels are available at a near-zero marginal cost, which pre-supposes a cislunar infrastructure of considerable complexity, the installation costs of which are not applied to energy production. As such the scope of this work is on "SPS number N", wherein the capital costs and non-recurring engineering costs are spread over a sufficient number of such that they are fully depreciated, and only new system costs need be considered. This permits an analysis of the LCOE of SSP assuming volume deployment, and thereby an indication of whether the new architecture presented here compares favorably with mature energy sources.

This is the author's manuscript of the article published in final edited form as: Schubert, P. J., Pires, B. C., do Nascimento, M., Barks, E., Nderitu, J., Goncalves, G., \& Tokmo, F. (2015). Analysis of a Novel SPS Configuration Enabled by Lunar ISRU. In AIAA SPACE 2015 Conference and Exposition (p. 4648). http://dx.doi.org/10.2514/6.2015-4648 


\section{Methods}

The SPS configuration upon which this work is based consists of a hollow cylindrical shell with an outer surface of PV panels (Figure 1). A central spire provides mechanical support to the slowly-rotating shell, and provides a conduit for the transfer of electrical power to a transmitting antenna. The "spacetenna" is a circular, phased-array device having a center of mass coincident with the central spire's long axis, and rigidly affixed to it. The entire assembly rotates at the same rate as the earth turns so that, in geostationary earth orbit (GEO) the spacetenna stays approximately aimed at a dedicated receiving antenna ("rectenna") on the earth's surface. The sun illuminates one side of the shell so that as it turns, each panel receives power for approximately one-third of each day. This arrangement presents an elegant solution for three significant challenges found in some prior SPS designs. First, there is no need to independently manipulate the sun-facing and the earth-facing portions of the design. This eliminates the need for a high-power slip ring, or other complex mechanism, to transfer power from the PV panels to the spacetenna. Second, heat rejection is built into the design whereby the hot backside of those panels facing the sun are exposed to the cold backside of those panels facing away from the sun. The cold panels transfer heat through their tiny thickness and radiate waste heat into the far reaches of outer space. Heat generated by the spacetenna radiates through the hollow cylinder, aided by heat pipes and thermoelectric devices which convey heat to cold PV panels. Third, this arrangement requires no careful alignment of any part of the structure. The PV-panelled shell can sway many degrees out of alignment with negligible loss of power, and the phased-array spacetenna can adjust its beam direction using phase shifters to maintain a steady

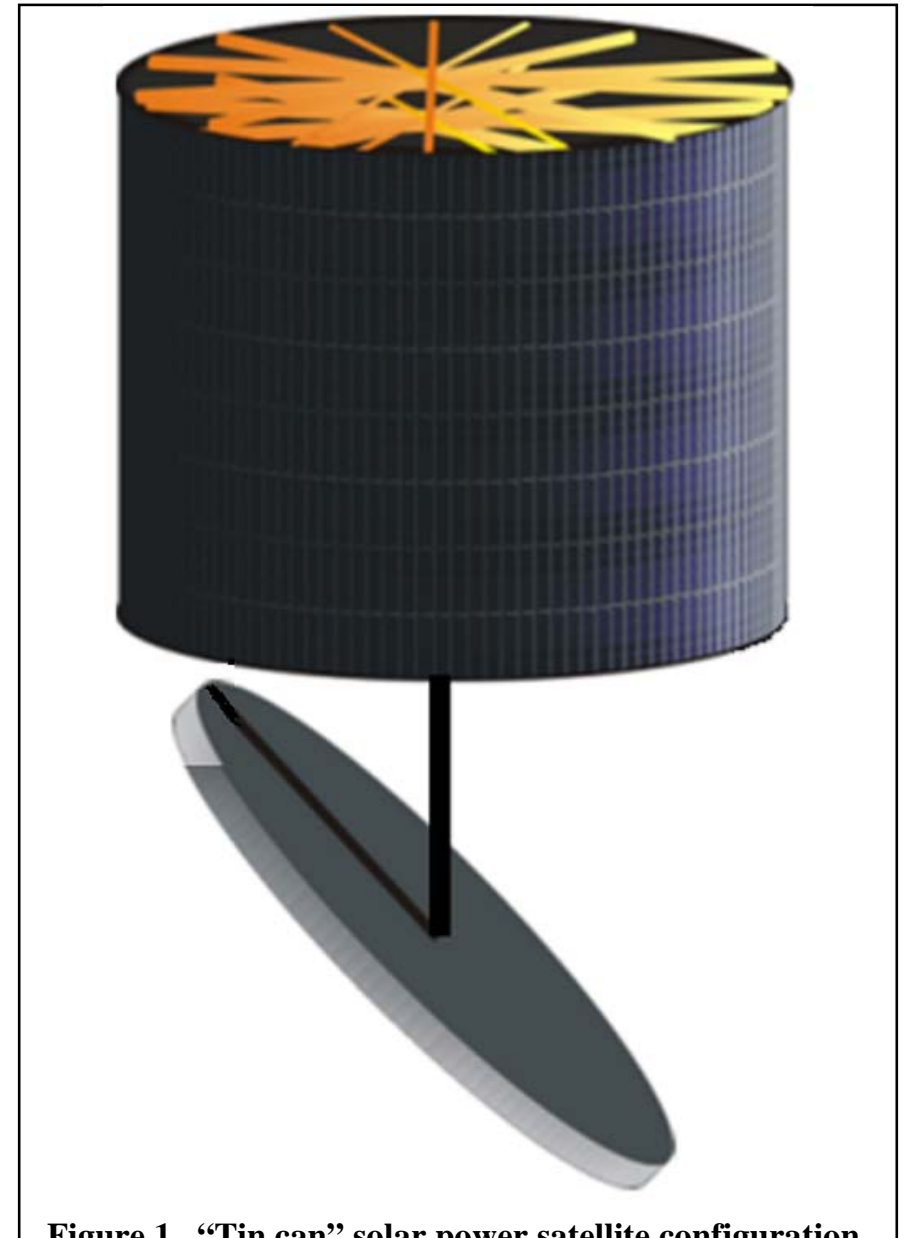

Figure 1. “Tin can" solar power satellite configuration. beam on the rectenna. Such a configuration, called the "tin can" design, thus has no moving parts ${ }^{5}$ !

\section{A. PV Generation Shell}

SPS number $\mathrm{N}$ is sized to gather 10 gigawatts $(\mathrm{GW})$ of electrical power. The lunar-sourced PV panels are single crystal silicon and have an assumed conversion efficiency of 22 percent. A silicon dioxide protective and anti-reflective layer is assumed with a refractive index of 1.46 so that sunlight impinging at angle greater than the critical angle from perpendicular is totally reflected. Within that $+/-75$ degree span, the amount of solar irradiation is equal to the solar constant of $1346 \mathrm{~W} / \mathrm{m}^{2}$ multiplied by the cosine of the angle between the sun and the individual PV panel. On average, this is equivalent to $25 \%$ of the entire outer surface being fully illuminated. Conversion of direct current (DC) power to radio-frequency (RF) energy is assumed to be 75 percent efficient so the area of illumination needed is 29 million square meters. This can be accommodated on a cylindrical shell which is $6.4 \mathrm{~km}$ in diameter and $6.4 \mathrm{~km}$ in height. The total energy incident on the shell is about $60 \mathrm{GW}$, so with $10 \mathrm{GW}$ collected, there is $50 \mathrm{GW}$ of thermal energy which must be rejected. The power management and distribution, and thermal management of this configuration are presented in the Results section.

Square PV panels of 0.167 meters side length are the building blocks for this SPS ${ }^{6}$. These arrive from the moon in spherical iron payload canisters in which the interstices are filled with refractory slag from the silicon separation process. This slag is used for ablative propulsion ${ }^{7}$ for initial spin-up, as well as for on-going station keeping. PV panels are arranged into square frames having a side length of 3.6 meters. The frame is made from the iron of the payload canister and is extruded from a solar-powered smelter. Additional fasteners are also fashioned from melted 
iron. Aluminum available from the moon is used for making electrical connections within frames, assembling slotted waveguides, and for conveying DC power to the central spire. Details of these fittings are presented in the Results section.

\section{B. Spacetenna}

The mass centroid of the phased-array spacetenna is coincident with the axis of symmetry for the cylindrical shell to minimize wobbling of the entire structure. The spacetenna is kept nominally oriented towards the earth, with a nominal angle approximating the lattitude of the rectenna. Rotation period is 24 hours. This generates centrifugal acceleration of $1.7 \mathrm{E}-5 \mathrm{~m} / \mathrm{s}^{2}$ for the PV generation shell, helping to preserve its shape while placing minimal demands on the guy wires holding it together.

In this work a transmission frequency of $2.45 \mathrm{GHz}$ is assumed. Element spacing is taken at 0.8 times the wavelength to avoid grating lobes. Drawing from the 1978 Reference Design ${ }^{8}$ the spacetenna is approximately $1 \mathrm{~km}$ in diameter and has a radial distribution of power following a Gaussian profile with edge taper of $-10 \mathrm{~dB}$ relative to the center. A pilot beam from the rectenna is assumed so that flatness of the spacetenna is less critical, with a receiver in each sub-array of 10 meters square.

Solid-state power amplifiers are assumed, based on wide bandgap semiconductors such as silicon carbide or gallium nitride. These must be assembled on earth, so terrestrial launch costs must be included for all such complex components. The power amplifiers deliver their signal to slotted waveguides made from lunar aluminum, and the RF energy emits from said slot.

\section{Rectenna}

The rectenna for a 2.45 $\mathrm{GHz}$ pencil beam from GEO is very large. The nominal circular beam diameter is 7.5 $\mathrm{km}$, and will expand into an ellipse depending on the lattitude, and on any difference in longitude from that of the SPS itself. The rectenna will array across the land, and consist of individual segments oriented nominally towards equatorial GEO. Figure 2 shows a rectenna having dipole elements mounted in front of a reflecting backplane. The backplane consists of suspended wires having a spacing less than one-half wavelength. For the 0.124 $\mathrm{cm}$ wavelength assumed here, this implies that at least $80 \%$ of the structure is open,

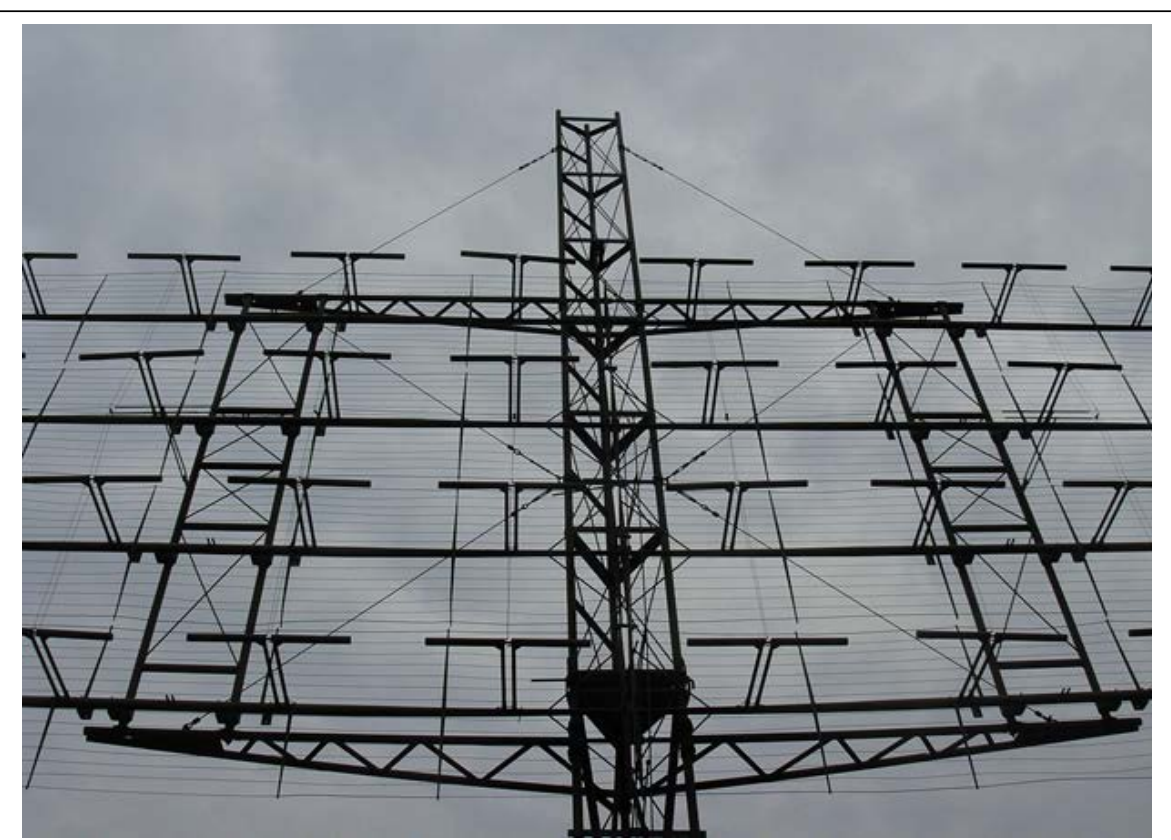

Figure 2. Phased-array receiving antenna showing dipole elements (split Tshapes) and backblane (horizontal wires).

and can admit sunlight. This makes it possible for other productive uses of the land underneath the rectenna, such as grazing or growing crops. A study was made exploring the tradeoffs between mounting the rectenna segments directly on the earth versus mounting them on poles at various elevations conducive to operations beneath. The higher the elevation (a maximum of 9 feet was considered) the greater the potential income from land use, but the more expensive the rectenna installation. It should be noted that the environmental footprint of the rectenna is not insignificant, as it will require a large amount of structural steel to support the rectenna elements regardless of their elevation.

Each T-shaped dipole element contains low-pass filters and a low turn-on voltage rectifying diode, which then produce DC power. The gathering of, and transmission of this power from across the rectenna is studied in the Results section with the aim of producing transmission lines for a nearby urban or industrial center. 


\section{Results}

\section{A. Cylindrical shell structural and thermal configuration}

The SPS tin can is an open cylindrical shell approximately 6.4 kilometers in both diameter and height. The shell surface is comprised of square iron frames, eaching having side lengths of 3.6 meters. The frames will be fabricated in orbit from the spherical shells of payload canisters arriving from the moon. T-shaped beams are formed via extrusion from a solar smelter. Beams are cut to length and welded together. Each frame holds approximately 460 PV panels that will be linked to one another using iron clips also manufactured by extrusion in zero gravity. As shown in the image below, the clip can be easily installed by simply sliding it on, and removed by applying pressure to the central ridge with a robotic system. This pressure will likely cause the clip to deform after one use, but it can be recycled for use in ongoing manufacturing processes. The PV panels will have a raised bump of aluminum on the top surface near the edges as a mechanism for the clip "teeth" to grasp onto, while also serving as one leg of the electrical contact. The frames themselves will be connected to the central spire with polymer cables shipped from earth (e.g. $\left.\operatorname{Kevlar}{ }^{\circledR}\right)$. Four short cables will be attached to hooks near the four corners of each frame, which will then be joined to the primary cable extending the remanding 3.2 kilometers to the central spire of the tin can structure. The end of each cable will be bent into a loop shape and crimped together, so that it may be easily connected to hooks on both the central spire and PV array frames. The thickness of the Kevlar cable required to support the frames under centripetal acceleration can be calculated by comparing the tensile load of the frame under rotation plus half the weigth of the cable itself, with maximum stress taken at 10 percent of the yield value. This is captured in Equation (1) where the result is a cable thickness of at least 3.2 microns. Variables are the number of panels $\left(\mathrm{Num}_{\mathrm{p}}\right)$, length and thickness and density of panels $\left(l_{\mathrm{p}}, \mathrm{t}_{\mathrm{p}}, \rho_{\mathrm{si}}\right)$, length of frame, cross sectional dimensions and density $\left(\mathrm{L}_{\mathrm{f}}\right.$, values shown, and $\left.\rho_{\mathrm{Fe}}\right)$, length and density of the cable $\left(\mathrm{l}_{\text {cable }}, \rho_{\text {cable }}\right)$, and maximum yield stress $(\sigma)$.

(1) $r_{\text {kevlar }}=\sqrt{\frac{\left(\left(\mathrm{Num}_{p} \times \mathrm{l}_{p}^{2} \times t_{p} \times \rho_{s i}\right)+\left(4 \times L_{f} \times((.0125 \times .0025)+(.01 \times .0025)) \times \rho_{F e}\right)\right.}{\pi\left(\frac{\sigma}{10}-\left(a \times \frac{1}{2} l_{\text {cable }} \times \rho_{\text {cable }}\right)\right)}}$

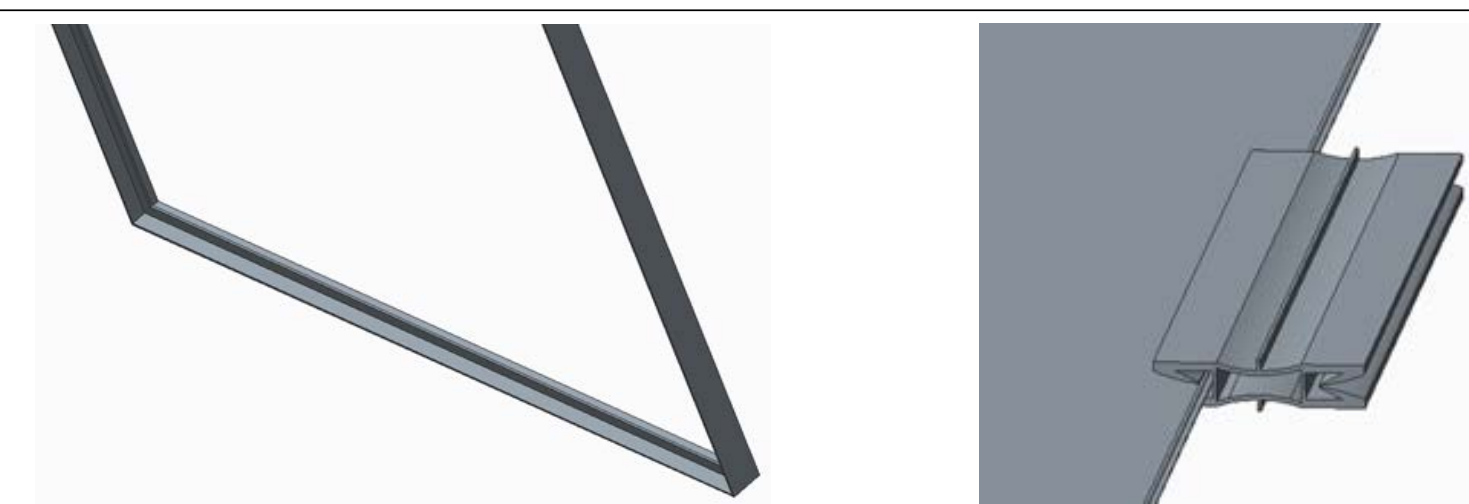

Figure 3. Frame (left) and clip (right) to hold individual solar panels

An important problem for a SPS is thermal management: $50 \mathrm{GW}$ which must be rejected radiatively. Appproximately $25 \%$ of the exterior surface of the cylindrical shell gathers solar heat. This heat rapidly transfers to the backside of the PV panels where it can radiate towards those PV panels presently in the dark. The view factor can be found by integrating differential areas for the sunlit and for the deep space-facing areas. Equation (2) shows the equation to be solved, where $\theta$ is the angle from the diameter to the hot side element (internal to cylinder) and $\phi$ is the angle from the diameter to the cold side, where theta is bounded on $-75,+75$ (due to critical angle), and phi is bounded on -90 and +90 to simplify the math, and ignore glancing angles to places maybe not so cold (this is a conservative assumption). The denominator is the square of the distance between differential patches for view factor, and $\theta_{1}$ and $\theta_{2}$ are the angles normal to those patches. 
(2) $F_{1 \rightarrow 2}=\frac{1}{A_{1} \pi} \iint \frac{\cos \left(\frac{-\theta}{2}+\frac{\phi}{2}\right) \cos \left(\frac{-\phi}{2}+\frac{\theta}{2}\right) \cos \left(\frac{l_{C}-l_{H}}{2 r}\right)}{r^{2}(2+2(\cos \theta \cos \phi+\sin \theta \sin \phi))+(l h-l c)^{2}} d A_{1} d A_{2}$

The view factor $\mathrm{F}_{1-2}$ integrates numerically to 0.69 . A solar panel with a anti-reflection coating and $22 \%$ conversion efficiency should have a value of $\varepsilon=0.78$. From the Stefan-Boltzmann law, balanced with the solar constant $\left(1356 \mathrm{~W} / \mathrm{m}^{2}\right)$, this translates to a temperature of $430 \mathrm{~K}$. If the darkside is exposed to deep space at $3.1 \mathrm{~K}$, then the temperature balance at the non-illuminated portion of the SPS can be calculated by setting equal the StefanBoltzman law for the sunlit and dark surfaces. This result is $393 \mathrm{~K}$. Thus, both illuminated and dark solar panels operate at the upper end of reasonable temperature ranges for silicon photocells (120 C to $157 \mathrm{C}$ ).

\section{B. SPS power management and distribution}

Along the perimeter, the solar panels will be strung together in two sets per ring, resulting in a voltage of 615.9 $\mathrm{kV}$ and a current of $16.56 \mathrm{~A}$ per string. This power will then be carried to the central spire using lunar aluminum wire, where it is condensed using custom combiner boxes that keep the positive and negative lines separate to avoid issues due to the high voltage. These combiner boxes will feed into one of 10 different copper bus bars ( 5 for positive and 5 for negative) that will take the power down the central spire to the spacetenna. HVDC cables were also researched to see if they were viable for use transmitting power down the central spire but it would have been extremely difficult to feed all the strings into a single wire. The busbars will feed into 5 HVDC transformer converter units that will convert the power to AC and then step down the power to acceptable levels. From here, the power will be transmitted to each transmitting antenna unit, of which there are 2.19 million elements. A $10 \mathrm{~W}$ power amplifier (PA) will serve 4.7 central antenna elements, but 46 at the periphery, for an average of 34 among the 74.9 million slotted waveguides. At each PA there will be an integrated inverter to convert the AC power back to DC.

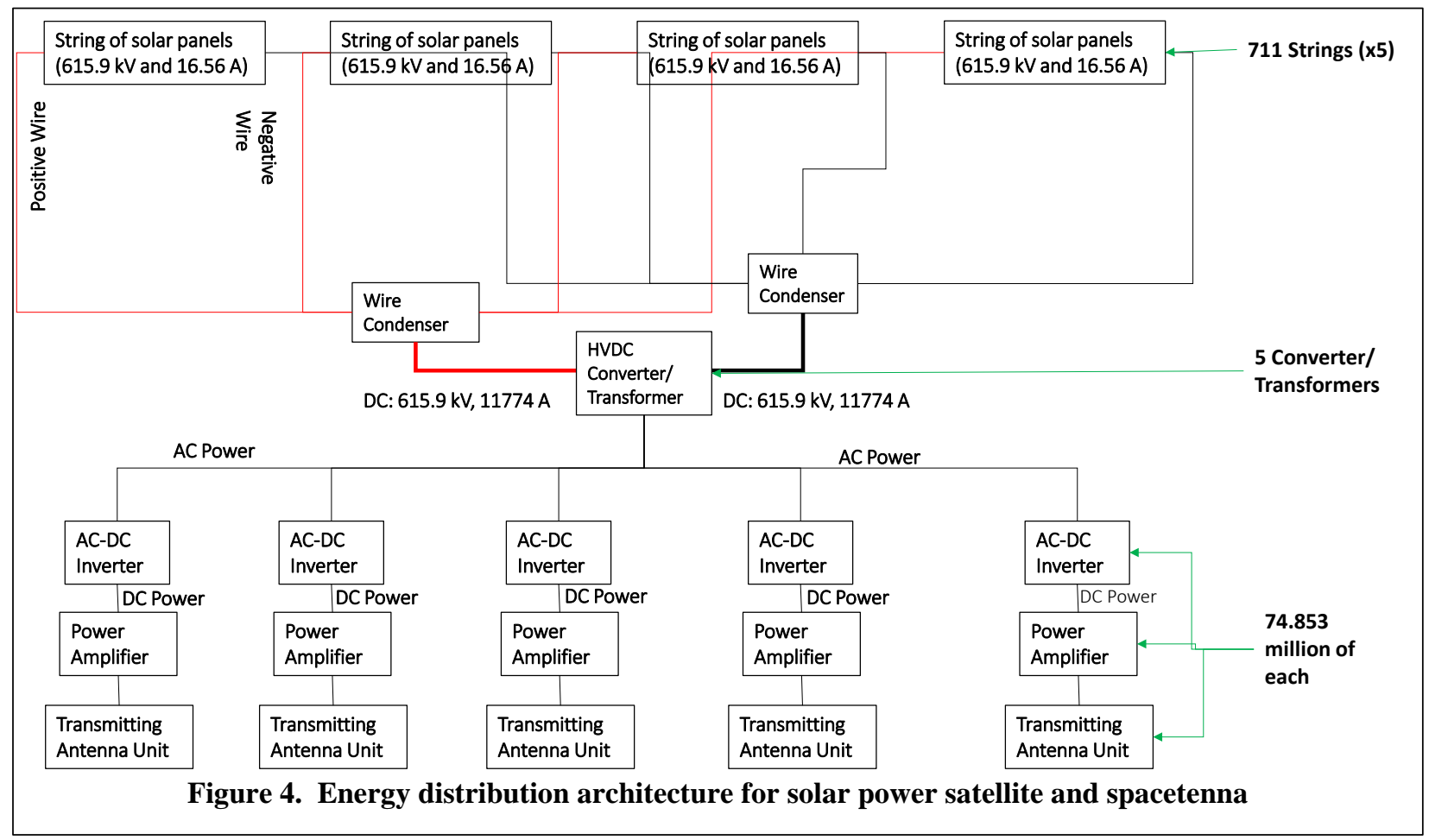

PAs have conversion efficiencies exceeding 90 percent $^{9}$, so that approximately $1 \mathrm{GW}$ of the nominal $10 \mathrm{GW}$ delivery must be rejected radiatively. As the SPS orbits the earth, the spacetenna is inclined about 23 degrees from the sun, and revolves once per day relative to it. Spreading 1 GW over a 945 meter diameter spacetenna is 1426 $\mathrm{W} / \mathrm{m}^{2}$. Adding sunlight heat on the spacetenna, at the cosine of 23 degrees and dividing by 2 to account for timeaveraging is an additional $624 \mathrm{~W} / \mathrm{m}^{2}$. Spread across the spacetenna this yields a temperature of $245 \mathrm{C}$ with no dissipation, which is certainly hot for electronics, yet within the range of performance for wide bandgap semiconductor solid-state power amplifiers. Radiators mounted on the opposite surface from the transmitting 
antennas can be positioned so that they radiate and reduce this high temperature somewhat, allowing for longer lifetime for components. Heat pipes may also be used to distribute heat from the higher-power PAs at the center of the spacetenna to the outer reaches. Water as a working fluid is useful from 5 to $230 \mathrm{C}$, and in combination with diamond-like carbon or other high thermal conductivity materials can even out the heat.

\section{Beam-forming}

One of the biggest challenges for the transmission of solar space power is to reduce the sidelobes surrounding the main power beam. High sidelobe levels could cause interference with other applications and also represent power losses in undesirable directions. This problem can be solved using two superimposed power beans, one half wavelength apart in the direction of propogation, in order to suppress the sidelobes from the transmitting antenna that are closer to the earth, and consequently avoid any interference with in terrestrial communications systems.

The first step of this project was to simulate the pattern for the antenna. For this purpose a Dolph-Chebyshev side lobe tapering technique was used, with a sidelobe level of $-30 \mathrm{~dB}$. After the simulation of the main beam, the procedure to reduce the sidelobes levels was to adjust the gain of the secondary beam to match the sidelobes power levels. However, the angular distribution of the secondary beam does not perfectly match with the primary beam, as seen in the top plot of figure 5. An innovative solution to this challenge was to vary the spacing between the elements in the secondary antenna, and consequently adjust the spatial distribution of the sidelobe in specific points of the curve close to the main beam. This is shown in the bottom plot of figure 5 .

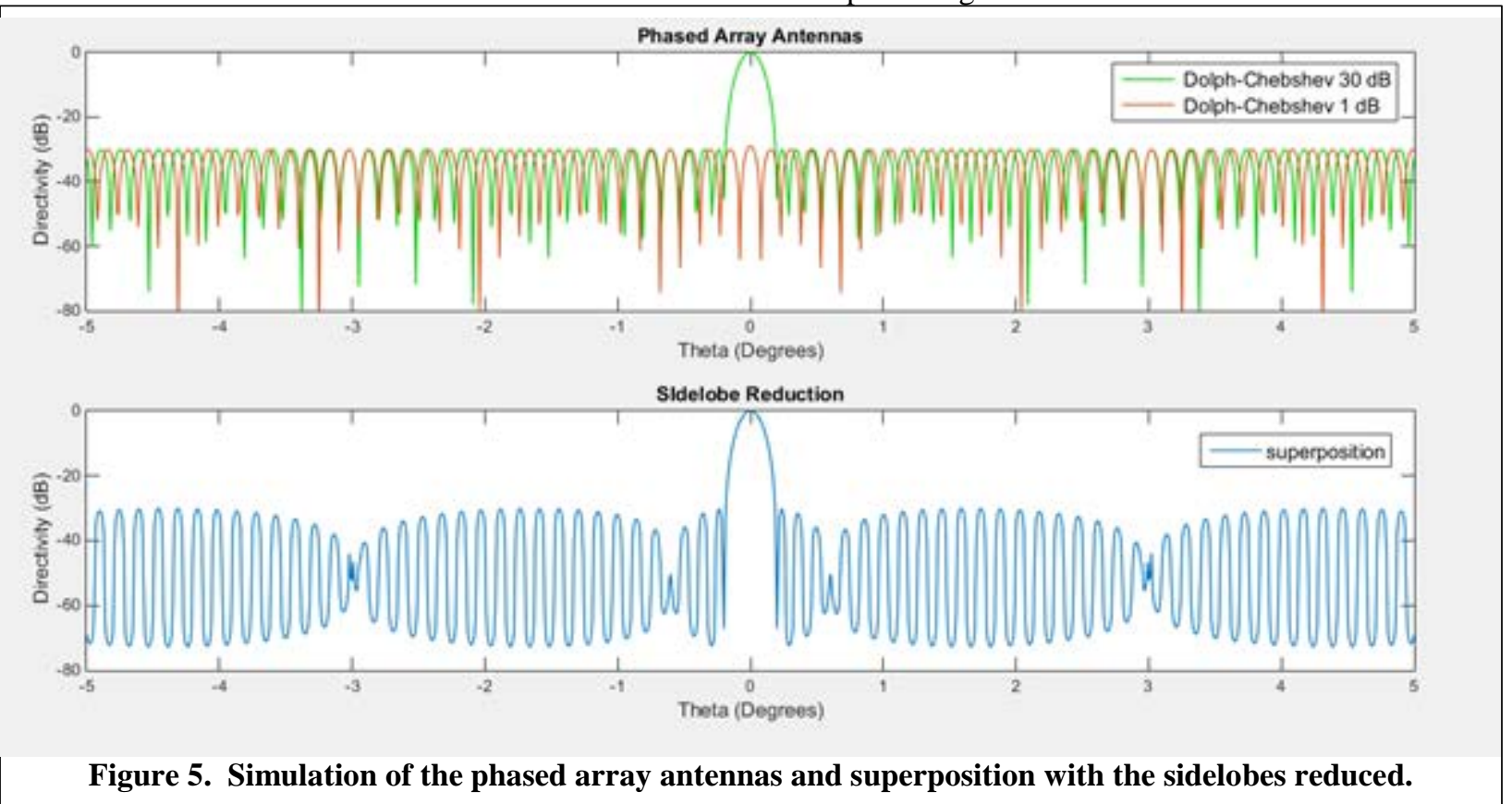

These results are preliminary, but point to the possibility that further refinement could afford additional improvements in sidelobe intensity shaping. It is unlikely that all sidelobes can be eliminated. But the earth is a fairly small target from GEO, so if the close-in sidelobes (within about 7 degrees) can be reduced, then interference with terrestrial communications may be minimized.

\section{Rectenna siting and power consolidation}

For a rectenna being served by a GEO SPS above the equator, the rounded surface of the earth distends the circular beam into an ellipse on the ground. For a $2.45 \mathrm{GHz}$ system the beam diameter from the reference case is 7.34 kilometers, and when delivered to 38 degrees latitude, has a major axis of $9.3 \mathrm{~km}$. The total area of the rectenna is $217 \mathrm{~km}^{2}$. This is sufficiently large that flat ground is not practical. Instead the rectenna is divided into square sections which are 3.6 meters on a side, approximately the size of ground-mounted solar panels. Because rectennas for $2.45 \mathrm{GHz}$ pass $80-90 \%$ of sunlight through their mesh, it is possible to consider alternative land uses for the area underneath. Three scenarios were considered: (a) mounting the sections three feet off the ground, with barely enough room to mow underneath, and no other land use possible; (b) six feet off the ground to permit cattle 
grazing; and (c) nine feet off the ground to permit tractors and combines to operate row crop agriculture. Snow loads and mechanical loads are assumed to be small, but wind loads must be considered. Equation (3) is used to compute wind loads, where $\mathrm{q}_{\mathrm{z}}$ is the velocity pressure coefficient, $\mathrm{K}_{\mathrm{z}}$ is the pressure exposure coefficient $(0.85), \mathrm{K}_{\mathrm{zt}}$ is the topographical factor (0.1), $\mathrm{K}_{\mathrm{d}}$ is the wind directionality factor (0.85), $\mathrm{I}=1$, and $\mathrm{V}$ is the maximum wind speed, taken to be 85 miles per hour. This coefficient is multiplied by the cross sectional area of the rectenna section to give the lateral force on the post upon which the section is mounted.

$$
q_{z}=0.00256 \times K_{z} \times K_{z t} \times K_{d} \times V^{2} \times I
$$

The maximum force is found to be $114 \mathrm{lb}$, and from this the dimensions, weight, and cost of the mounting post can be calculated using the bending stress for a cantilevered beam with a load at the end. A square post is assumed, made of structural steel, and a 10x safety margin is used from the maximum yield strength. Bending stress $(\sigma)$, moment (M), distance from neutral axis to edge of member (Y), and moment of inertia (I) are shown in equation (4), where $\mathrm{B}=\mathrm{H}$ are the cross sectional side lengths, $\mathrm{L}$ is the height of the post, and $\mathrm{F}$ is the force found above.

$$
\sigma=\frac{M . Y}{I} \quad M=F x L \quad Y=\frac{\mathrm{B}}{2} \quad I=\frac{B H^{3}}{12}
$$

Susbstituting the latter three equations into the first, and setting the maximum stress to $25 \mathrm{MPa}$, the cross section side lengths for each post height above are: (a) 1.9 inches for 3 feet; (b) 2.4 inches for 6 feet; and (c) 2.73 inches for 9 feet. Assuming a solid post, and multiplying by a factor of 2 for the ground screw footer into which the post is mounted, the total mass of steel can be computed for each case, for a total of 27.2 million sections. For each scenario, the mass of steel in tons is: (a) 241,000; (b) 299,000; and (c) 510,000 for 9 feet of height. Steel prices are included in the economic calculations presented below. Land prices for the 53 thousand acres are about $\$ 63$ million if the land is used for grazing, and \$212 million if the land is used for row crops.

The dipole elements will first be connected in series to achieve the desired transmission voltage of $1.2 \mathrm{MV}$. The result will be a high voltage string of elements which will then be connected in parallel with other strings to provide power to HVDC cables rated at 200 amps. This method of connecting the elements is called 'series parallel'. Figure 6-left shows how the elements are connected in series to complete a string. Figure 6-right shows how the strings are then connected in parallel. This method is used throughout the entire rectenna and provides a simple way to connect the dipole elements and transmit the power to the receiving grid.

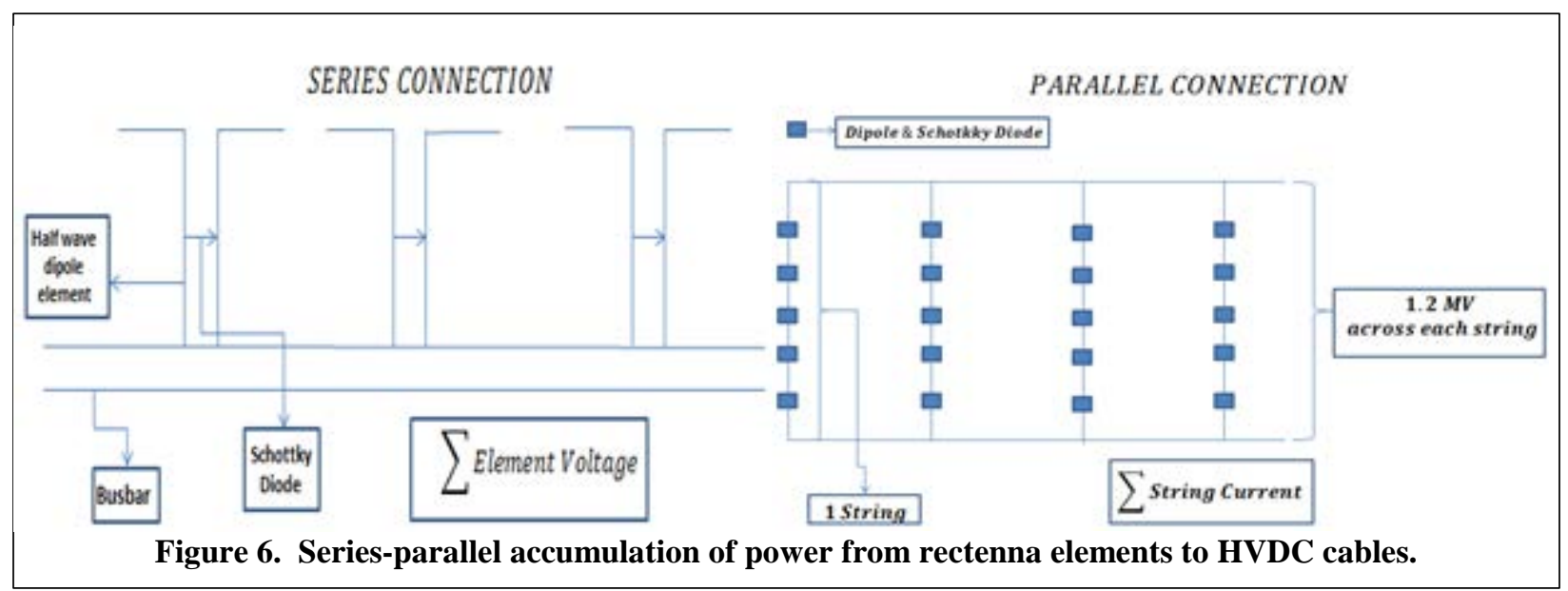

In our study the rectenna was divided into 25 regions, each radiating from the center like a pizza sliced into triangular pieces. Each region consists of the series-parallel connected elements. The goal was to produce a 200 ampere HVDC cable within each region. This was accomplished by connecting each of the elements so that the 200 ampere goal could be accumulated as it moved from the inner elements to the outer elements. After working outward; the result is a rectenna that has 25 HVDC cables rated at $1.2 \mathrm{MV}$ and pumping out 200 amps. This produces a total of $5.35 \mathrm{GW}$ with all efficiencies and ohmic losses taken into account (53.5\% DC-DC efficiency). 


\section{Analysis}

\section{A. Overall economics}

Two bold assumptions are made regarding economics. First, the cislunar infrastructure for moving materials electromagnetically ${ }^{4,6,7}$ is assumed to be pre-existing and have negligible operating costs. Second, a large-scale lunar manufacturing capability is extant, operated robotically, powered by sunlight, and can provide solar panels, aluminum, and iron with negligible cost. The cost of satellite number $\mathrm{N}$ is therefore dominated by the launch costs for SPS components which are too complicated for the lunar factories. This includes the following:

a) Monolithic microwave integrated circuit (MMIC) power amplifiers (PA) rated at $10 \mathrm{~W}$,

b) Copper bus bars to gather high voltage DC current from solar panels in the central spire,

c) HVDC transformer-converter units.

Mass of a PA is taken at $0.4 \mathrm{~kg}^{10}$, copper is 1900 metric tons, and the 5 transformers are 410 metric tons each. The total mass to launch is 4930 MT, and using the published cost estimates for Falcon Heavy to GEO amounts to $\$ 20.5$ billion. Procurement costs for launched components is $\$ 1.3 \mathrm{~B}$. The ground system consisting of the rectenna elements, mounting poles and ground screws, and HVDC cable to the edge of the owner controlled is $\$ 2.6 \mathrm{~B}$. The sum of these one-time costs for a mature operation is $\$ 24.4 \mathrm{~B}$ for a $5.35 \mathrm{GW}$ delivered power. The specific cost metric is $\$ 4.56 /$ Watt.

\section{B. Global thermal impact of power generation}

One concern with power beams through the earth's atmosphere is the amount of heat generated. From the reference study ${ }^{8}$ atmospheric losses of 2 percent are assumed. Considering only the trophosphere, taken at a mean altitude of $13.5 \mathrm{~km}$, and using air density and heat capacity at sea level, the total heating is then 0.02 times the power being delivered to earth. The average wind speed in the U.S. is $7 \mathrm{~m} / \mathrm{s}$, so the air within the columnar shape of the power beam will be refreshed in the time required for the air to pass through the diameter of the beam. Combining the standard equation for heating a mass with the "refresh time" of the air in the beam, we derive equation (5) for the steady-state increase in temperature of the air.

(5) $\Delta T_{\text {air }}=\frac{\left(\frac{1.887 \times 10^{-4} J}{s m^{3}}\right) x(1428.6 s)}{\left(\frac{1275.4 g}{m^{3}}\right) x\left(\frac{1.0035 J}{g K}\right)}$

The result is an increase of air temperature of $2.1 \times 10^{-4} \mathrm{~K}$ from a SPS power beam. For comparison purposes a nuclear power plant of equivalent electrical output is considered. The owner controlled area around a nuclear power plant is approximately equal to that of a $2.45 \mathrm{GHz}$ power beam from GEO, so the air volumes are set equal between these two cases. Assuming the nuclear plant is 30 percent efficient and that half of the remaining thermal energy is released into the air, equation (5) can be used to compute an air temperature rise of $6.1 \times 10^{-3} \mathrm{~K}$, a value which is some 30 times higher for the nuclear plant relative to the SPS power beam.

\section{Conclusions}

Presented herein is an architectural and systems study of a novel design for a solar power satellite. This work addresses structural configuration and assembly methods, power management and distribution, power beaming and sidelobe reduction, plus rectenna layout and power accumulation. Approximate costs are provided for a mature infrastructure based on lunar-source materials for solar panels and structural components of the powersat, and the impact on atmospheric heating is analyzed and compared to a nuclear power plant of comparable size. The results are based on critical assumptions that the infrastructure has been fully depreciated and indicates the potential for a specific cost of \$4.56 per Watt of capacity. The surfeit of solar panels provides integral thermal management and also reduces the damage from solar wind, so the panels may be expected to survive an average of 15 years. Assuming a 97 percent uptime and operations and maintenance costs which are far below the system cost of \$24.4B this yields a levelized cost of energy (LCOE) of \$36 per MWh, which is approximately equal to low end of the range for utility scale wind farms (on-shore) and is appreciably lower than the 2014 ranges for terrestrial PV at utility scale (\$72-86/MWh). This novel method of SSP is also less expensive than conventional sources of cheap energy, for instance coal-fired power plants (\$66-151/MWh), nuclear power plants (\$90-134/MWh $)^{11}$. The economic analysis ignores the additional costs for sidelobe reduction, which is much lower in power than the primary load and expected to be a minor contribution to launch costs. The copper lofted into orbit could be replaced by aluminum from the lunar surface, which would lower the cost to $\$ 16.3 \mathrm{~B}$ but would require considerably more infrastructure both on the moon and in orbit. Another option is to use wireless power transfer down the central spire 
of the SPS to save launch mass. Further, it may be possible to design large-scale buck converters to maintain DC power throughout the SPS, avoiding 2 extra conversions. Each of these topics is expected to be the subject of further study.

Land use was addressed above, but it may also be possible to float the rectenna in seawater. This has appeal in that orbital slots for GEO constellations are crowded especially over large north-south landmasses. An auxiliary benefit for a sea-sited rectenna is the ability to produce desalinated water during periods of low demand (e.g. at night). In many areas of the world, water is as important as power so that providing both addresses two important social needs.

\section{References}

${ }^{1}$ US Energy Information Agency, http://www.eia.gov/electricity/wholesale/, accessed 11 Aug. 2015

${ }^{2}$ Burton, R.L., Schubert, P.J., Rysanek, F., Fenoglio, P., Hutches, P., "Oxygen Extraction Apparatus and Process," US 7,935,176, 3 May 2011.

${ }^{3}$ Schubert, P., “Solar Panels from Lunar Regolith,” Int'l. Space Development Conf., Chicago, IL, 28-31 May 2010.

${ }^{4}$ Duncan, S., Schubert, P., Delaurentis, D., "System for Electromagnetic Capture of Lunar-launched Payloads into GEO,” 43rd AIAA/ASME/SAE/ASEE Joint Propulsion Conference, Cincinnati, OH 8-11 July 2007.

${ }^{5}$ Schubert, P.J., "Energy Resources Beyond Earth - SSP from ISRU," Gateway to Space, St. Louis, MO, 7-9 Nov. 2014.

${ }^{6}$ Schubert, P., Simpson, N., Lin, M., “Technical Feasibility of a Novel Method for Station Keeping,” AIAA SPACE 09, Pasadena, CA, 14-17 Sept 2009

${ }^{7}$ Schubert, P.J., "System and Method for Attitude Control and Station Keeping,” US 7,118,075, 2006.

${ }^{8}$ Department of Energy and NASA, "Satellite Power System Concept Development and Evaluation Program, Reference System Report,” Oct. 1978.

${ }^{9}$ Schmelzer, D., Long, S.I., “A GaN HEMT class F amplifier at $2 \mathrm{GHz}$ with > 80\% PAE,” IEEE J. Solid-State Circuits, v. 42, no.10, p. 2130-2136, Oct. 2007.

${ }_{11}^{10}$ Dr. Ho Huang, AMCOM, personal communication, 11 Aug 2015.

11 "Lazard's Levelized Cost of energy Analysis - Version 8.0," September 2014, http://www.lazard.com/media/1777/levelized_cost_of_energy_-_version_80.pdf, accessed 6 Feb 2015. 